\title{
SORTING OF MESOSCOPIC PARTICLES DRIVEN THROUGH PERIODIC POTENTIAL LANDSCAPES
}

\author{
A.M. LACASTA \\ Departament de Física Aplicada, Universitat Politècnica de Catalunya, \\ Avinguda Doctor Marañon 44, E-08028 Barcelona, Spain \\ anna@fa.upc.edu \\ M. KHOURY and J.M. SANCHO \\ Departament d'Estructura i Constituents de la Matèria, Facultat de Física, \\ Universitat de Barcelona, Diagonal 647, E-08028 Barcelona, Spain \\ KATJA LINDENBERG \\ Department of Chemistry and Biochemistry 0340 and Institute for Nonlinear Science, \\ University of California, San Diego, La Jolla, California 92093-0340, USA
}

\begin{abstract}
Sorting of colloidal particles of different sizes is of importance in the transport and delivery of such particles in biological, materials science, and other technological contexts. A successful methodology involves the flow of a mixture of particles over designer surfaces presenting a periodic array of traps (optical tweezers) or microfabricated obstacles. The trajectories of the particles over these surfaces deviate from the direction of flow as the particles are attracted (traps) or repelled (obstacles) by the features of the landscape. The deviation of the particles from that of the flow depends on particle size (or on some other particle characteristic) and hence the particles can be sorted according to trajectory direction. On the basis of extensive numerical simulations, we present a unified view of these methodologies and discuss the effects of system parameters such as the magnitude and direction of the flow on the sorting efficacy.
\end{abstract}

Keywords: sorting; colloidal particles; periodic potential landscapes

\section{Introduction}

In the past few years there has been a veritable burst of effort and success in technologies that lead to efficient and nondestructive sorting of mixtures of colloidal particles, most of the interest in this capability arising in biological contexts. One particular approach that has attracted a great deal of attention is that of separating particles by having them flow over a surface modulated with traps and/or obstacles whose spatial distribution causes an angular deviation of particles from the direction of the flow. ${ }^{1-14}$ The magnitude and direction of the angular deviation depend on the geometry of the surface and, most importantly, on some particle property such as its size or index of refraction, thus making it possible to separate particles because 
they emerge at different angles. Trap landscapes have been generated with optical tweezers, ${ }^{3-8,14}$ and obstacle landscapes with microfabricated technology. ${ }^{9}, 10$

The sorting efficiency of these devices depends on a number of details of the experiment including surface structure and geometry, the flow speed of particles over the surface, the particular particle characteristic used for the separation process, interactions among particles, thermal fluctuations, etc., and a good deal of work has gone into optimizing conditions in each of the experimental approaches such as to improve the efficiency of sorting. This is, of course, the important course as a basis for manufacturing devices for different applications. On the other hand, at a proof-of-principle level it is important to remember the common aspects of the various methodologies, and to keep in mind that it is useful to extract and organize the generic features that lead to the desired sorting. This is the purpose of our review.

While there are some analytic results in the literature, ${ }^{12,13,15}$ most of the theoretical understanding of the problem is based on numerical simulation results. $^{2,6,16,17}$ Our presentation here also focuses on numerical results, and is organized as follows. In Sec. 2 we present the basic model, discuss the variables that are most important for effective sorting, and present the various surfaces to be considered in this presentation. In Secs. 3 and 4 we display a variety of simulation results for particle trajectories and for figures of merit that lead to optimal parameter choices for effective sorting. In Sec. 5 we consider the effects of temperature and of parameters that have been kept constant in the remainder of the discussion. Finally, we summarize our results in Sec. 6.

\section{The Model}

Our analysis is based on straightforward classical equations of motion to describe the motion of a particle of mass $m$ on a two-dimensional surface,

$$
\begin{aligned}
& m \ddot{x}=-\frac{\partial}{\partial x} V(x, y)-\mu \dot{x}+f_{x}+\xi_{x}(t) \\
& m \ddot{y}=-\frac{\partial}{\partial y} V(x, y)-\mu \dot{y}+f_{y}+\xi_{y}(t) .
\end{aligned}
$$

The position of the particle is specified by the coordinates $(x, y)$, and a dot denotes a derivative with respect to time $t$. The modulation of the surface is described by a periodic potential $V(x, y)$ of height or depth $V_{0}$ and period $\lambda$ which for simplicity we take to be equal in the $x$ and $y$ directions. A constant external force with Cartesian components $f_{x}, f_{y}$ (magnitude $\left.f_{0}=\left(f_{x}^{2}+f_{y}^{2}\right)^{1 / 2}\right)$ acts on the particle. This force might model, for instance, a constant velocity flow. The Stokes coefficient of friction is $\mu$, and the thermal environment is captured by the mutually uncorrelated white noises $\xi_{i}(t)$ that obey the equilibrium fluctuation-dissipation relation at temperature $T$,

$$
\left\langle\xi_{i}(t) \xi_{j}\left(t^{\prime}\right)\right\rangle=2 \mu k_{B} T \delta_{i j} \delta\left(t-t^{\prime}\right)
$$


These equations are simplified by rescaling via the transformations of variables

$$
r_{x}=\frac{x}{\lambda}, \quad r_{y}=\frac{y}{\lambda}, \quad \tau=\sqrt{\frac{V_{0}}{m}} \frac{t}{\lambda}
$$

and by introducing the scaled potential of unit height or depth $\mathcal{V}=V / V_{0}$. In terms of these quantities

$$
\begin{aligned}
\ddot{r}_{x} & =-\frac{\partial}{\partial r_{x}} \mathcal{V}\left(r_{x}, r_{y}\right)-\gamma \dot{r}_{x}+F_{x}+\zeta_{x}(\tau), \\
\ddot{r}_{y} & =-\frac{\partial}{\partial r_{y}} \mathcal{V}\left(r_{x}, r_{y}\right)-\gamma \dot{r}_{y}+F_{y}+\zeta_{y}(\tau),
\end{aligned}
$$

where a dot now denotes a derivative with respect to $\tau$, and where the scaled white noise terms obey the fluctuation-dissipation relation

$$
\left\langle\zeta_{i}(\tau) \zeta_{j}\left(\tau^{\prime}\right)\right\rangle=2 \gamma \mathcal{T} \delta_{i j} \delta\left(\tau-\tau^{\prime}\right)
$$

In addition to lattice geometry and potential function parameters, the problem is defined by four independent (dimensionless scaled) parameters: the temperature $\mathcal{T}$, the dissipation $\gamma$, the magnitude $F_{0}$ of the external force,

$$
\mathcal{T}=k_{B} T / V_{0}, \quad \gamma=\mu \lambda / \sqrt{m V_{0}}, \quad F_{0}=\frac{\lambda f_{0}}{V_{0}},
$$

and the angle $\theta$ between the external force and the $x$ axis.

Although in all of our simulations we implement the full equations of motion (4), it should be noted that in the regime of interest in the sorting problem two simplifications are appropriate. One is that the dissipation parameter $\gamma$ is large, i.e., the system is overdamped, so that the second derivative term can be dropped entirely. This is of great help in any analytical work. ${ }^{15}$ In all of our simulations we set $\gamma=20$. The second is that sorting of particles is hindered by thermal fluctuations, and so the technology is useful precisely in the regime where thermal effects are unimportant. Therefore the temperature (noise) contributions could also be dropped from the equations. In other words, the problem in the parameter regimes of greatest interest reduces to a pair of first order purely deterministic equations. However, by retaining thermal fluctuations in our simulations we are able to show the extent to which these fluctuations affect the results. Still, this discussion serves to focus on the fact that the parameters of particular interest in this study are those that define the potential, and the magnitude $F_{0}$ and direction $\theta$ of the external force.

We begin by discussing the surface modulations, i.e., the potentials, that might be interesting. Surfaces constructed with arrays of optical tweezers usually consist of periodic arrays of identical localized traps or potential wells separated by flat plateaus. ${ }^{3-8}$ (Interesting variations of this basic technology to further improve performance are under active study. ${ }^{14}$ ) In our earlier work ${ }^{15-17}$ we mimicked this configuration with the potential (written in the original unscaled form)

$$
V(x, y)=\frac{-V_{0}}{1+e^{-g(x, y)}},
$$


where $g(x, y)$ is the periodic two-dimensional function

$$
g(x, y)=A[\cos (2 \pi x / \lambda)+\cos (2 \pi y / \lambda)-2 B] .
$$

The parameter $V_{0}>0$ controls the depth of the traps, $A$ controls the steepness of these features, and $B$ determines the relative size of a well with respect to the spatial period $\lambda$. In particular, larger values of $B$ lead to shallower and narrower wells and are therefore associated with larger particles, and subsequently we focus on the effects of this parameter as a sorting indicator. With a very occasional exception, throughout our simulations we set $A=5$, and (although it does not matter in the scaled representation) $\lambda=4$ and $V_{0}=1$. A finite portion of this two-dimensional potential is seen in the upper left panel of Fig. 1.
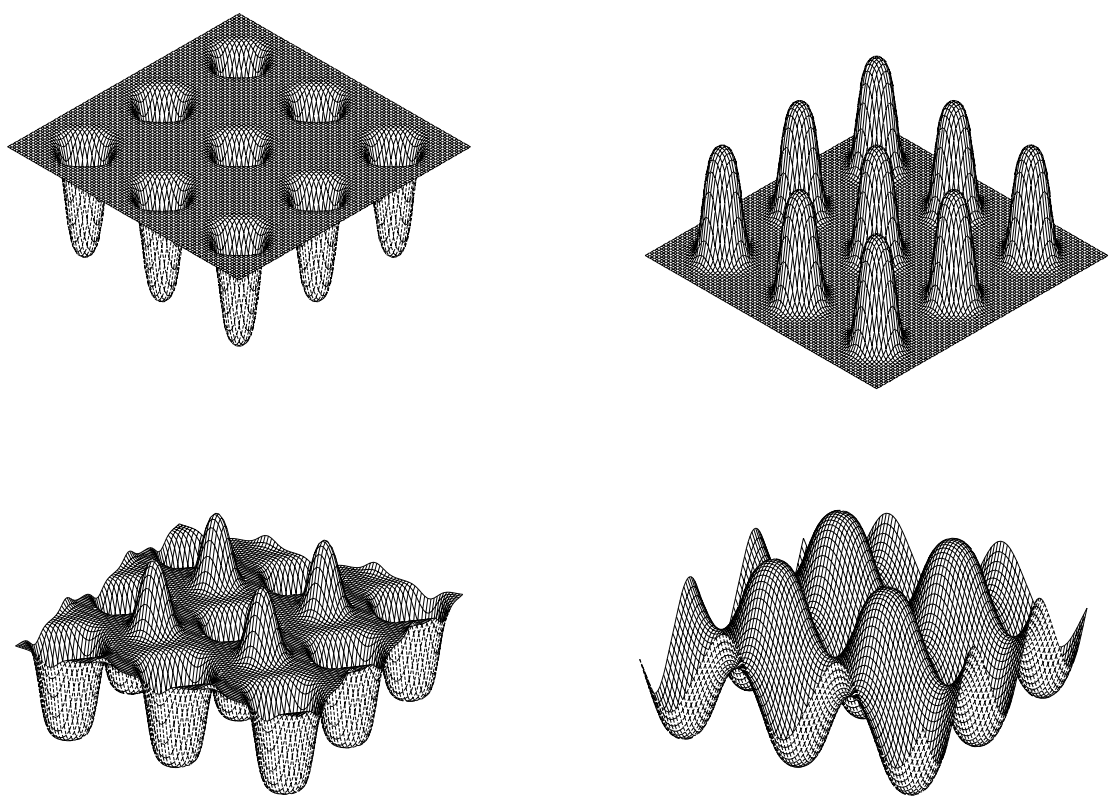

Fig. 1. Finite portions of typical two-dimensional potentials that lead to particle sorting. Upper left panel: periodic arrangement of traps with $B=0.7$. Upper right panel: periodic arrangement of obstacles with $B=0.7$. Lower panels: mixed arrangements of traps and obstacles. Lower left: $B_{W}=0.5$ and $B_{O}=0.9$. Lower right: $B_{W}=B_{O}=0.5$ and $A=2$.

A second modulation consists of regularly placed objects or barriers. ${ }^{9,10}$ These barriers can be shaped and inclined in different ways and placed on the surface in a variety of geometric arrangements that can be manipulated to increase their sorting efficiency, but a generic representation for numerical simulations can be achieved by simply flipping the potential (7), $V \rightarrow-V$. The parameter $V_{0}$ now controls the height of the obstacles, and larger values of $B$ lead to wider "corridors" between 
obstacles and are therefore associated with smaller particles. A finite portion of this potential is seen in the upper right panel of Fig. 1.

While surfaces with traps and surfaces with obstacles have been studied rather extensively, we add a third type of surface to our discussion that has not been realized experimentally (and may or may not be of particular experimental interest), but that helps to round out our understanding of the sorting capabilities of modulated surfaces. This is a surface in which there are both obstacles and traps periodically placed with respect to one another. For this purpose we simply generalize the potential to contain both, with the possibility of different "sizes" $B$ for the wells and obstacles,

$$
V(x, y)=\frac{-V_{0}}{1+e^{-g_{W}(x, y)}}+\frac{V_{0}}{1+e^{-g_{O}(x, y)}},
$$

where

$$
\begin{gathered}
g_{W}(x, y)=A\left[\cos (2 \pi x / \lambda)+\cos (2 \pi y / \lambda)-2 B_{W}\right] \\
g_{O}(x, y)=A\left[\cos (2 \pi x / \lambda+\pi)+\cos (2 \pi y / \lambda+\pi)-2 B_{O}\right] .
\end{gathered}
$$

The lower panels in Fig. 1 show two cases of this potential. The purpose of including the lower right case is to illustrate that one can choose parameters in the mixed potential to mimic a purely cosinusiodal potential.

In the physical experiments of interest, a stream of particles is made to flow over such a surface say starting at the origin of the $x$ axis, with a particular direction of flow that can be varied relative to the symmetry axes of the periodic lattice. Some distance downstream, say at $x=L_{x}$, the particles are collected and one observes the angle at which they emerge relative to the angle of flow. If this emergence angle is different for particles of different sizes (or some other particle characteristic), then the particles can be sorted as they emerge. As noted earlier, the important variables for sorting efficiency are the angle $\theta$ between the flow direction and the symmetry axis ( $x$ axis) of the lattice, the strength of the flow as reflected in the magnitude $F_{0}$, and the size of the particles as reflected in the potential parameter $B$.

Having discussed the models to be analyzed, we now focus on the particle trajectories in these various potential landscapes and the dependences of these trajectories and the resulting sorting capabilities on the important parameters. For this purpose we need to specify the observables to be calculated in our numerical simulations. Most are related to the mean particle velocity $\langle\boldsymbol{v}\rangle$ as a function of these parameters. More specifically, for any given potential, our numerical simulations yield the Cartesian components of the average velocity,

$$
\left\langle v_{i}\right\rangle=\lim _{\tau \rightarrow \infty} \frac{\left\langle r_{i}(\tau)\right\rangle}{\tau},
$$

from which we construct the components parallel and perpendicular to the force $\boldsymbol{F}$,

$$
\left\langle v_{\|}\right\rangle=\left\langle v_{x}\right\rangle \cos \theta+\left\langle v_{y}\right\rangle \sin \theta, \quad\left\langle v_{\perp}\right\rangle=-\left\langle v_{x}\right\rangle \sin \theta+\left\langle v_{y}\right\rangle \cos \theta .
$$


The magnitude of the deviation of the direction of the average particle velocity from that of the force can be characterized by either of the angles $\alpha$ (called the deflection angle) or $\Psi=\alpha+\theta$ (called the velocity angle), defined as

$$
\tan \alpha=\frac{\left\langle v_{\perp}\right\rangle}{\left\langle v_{\|}\right\rangle}, \quad \tan \Psi=\frac{\left\langle v_{y}\right\rangle}{\left\langle v_{x}\right\rangle} .
$$

\section{Trajectories: Dependence on $\theta$}
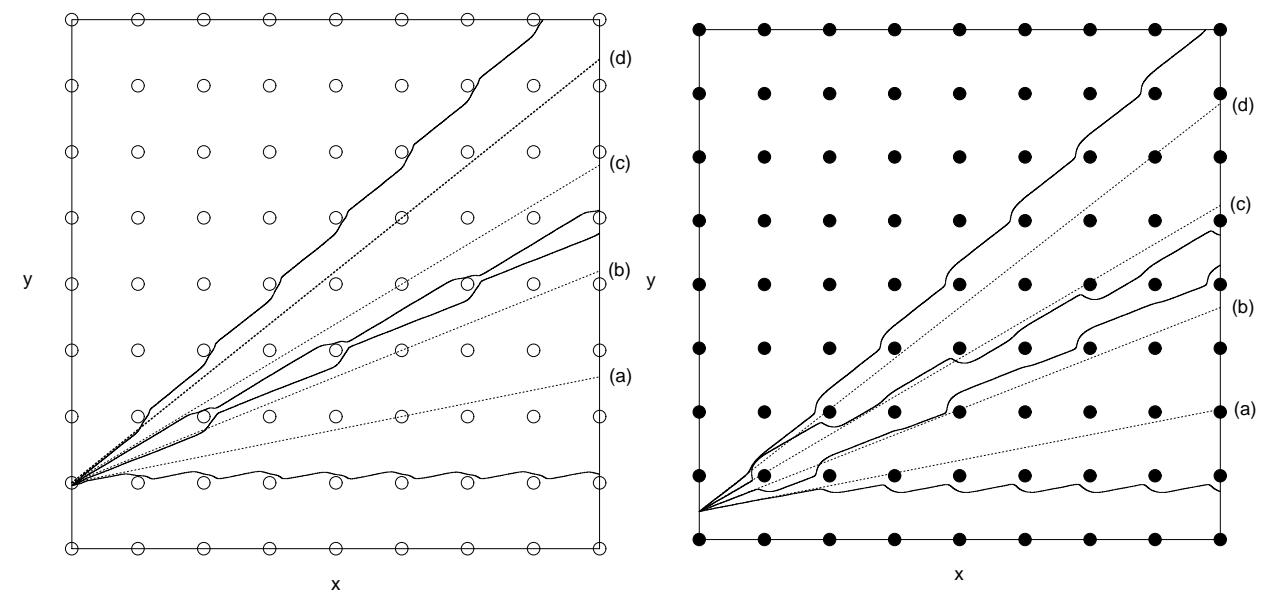

Fig. 2. Left panel: typical particle trajectories on a lattice of traps for forces applied at different angles represented by the dotted lines. Right panel: same for a lattice of obstacles. Potential parameters are those of the upper panels in Fig. 1 . Other parameters: $F_{0}=10, \mathcal{T}=10^{-4}$, and $\tan \theta=0.2(\mathrm{a}), 0.4(\mathrm{~b}), 0.6(\mathrm{c})$, and $0.8(\mathrm{~d})$.

Typical trajectories that illustrate the crux of the story are shown in Fig. 2. The left panel shows a lattice of traps (the same as in the upper left panel in Fig. 1) and a series of particle trajectories associated with different flow directions as indicated by the small letters on the side of the panel. The right panel shows a lattice of obstacles (the same as in the upper right panel of Fig. 1) and the trajectories in this lattice. The figures clearly show the geometrical source of the deviations of the particle trajectories from the flow. In the surface with traps, as particles move on the surface they are attracted to the nearest traps. Every time a particle moves close to a trap, there is a small pull away from the flow and toward the trap, leading to a net angular displacement between the two directions. In the surface with obstacles, the particles are repulsed by the nearest barriers. Every time a particle moves close to one, it must avoid it and as a result it is pushed away from the flow, again leading to a net angular displacement. Thus, in a sense, the deviation mechanism is "opposite" in the two potentials. There are, however, noteworthy points that are explored in more detail subsequently:

(1) While details of the trajectories in the two cases are different, the signs of 
the deflection angles $\alpha$ are the same even though one involves attractive and the other repulsive forces. While this may sound somewhat counterintuitive, the figure clarifies the process, cf. cases (a) and (d). For both potentials, $\alpha$ is positive for case (a) and negative for case (d). This result is supported analytically in Eq. (19) in Gleeson et al., ${ }^{15}$ where a perturbative analysis valid for large forces and temperatures explicitly shows the particle velocities to be independent of the sign of the potential.

(2) The range of angles covered by the emerging particles associated with a given range of flow angles may be narrowed in both geometries. Thus, for example, in both geometries all flows between direction (b) and (c) emerge within the much narrowed cone indicated in the figures. Again, the details are different (e.g. the emergence cone is much narrower for the wells than for the obstacles in this particular example), but the overall behavior is the same.
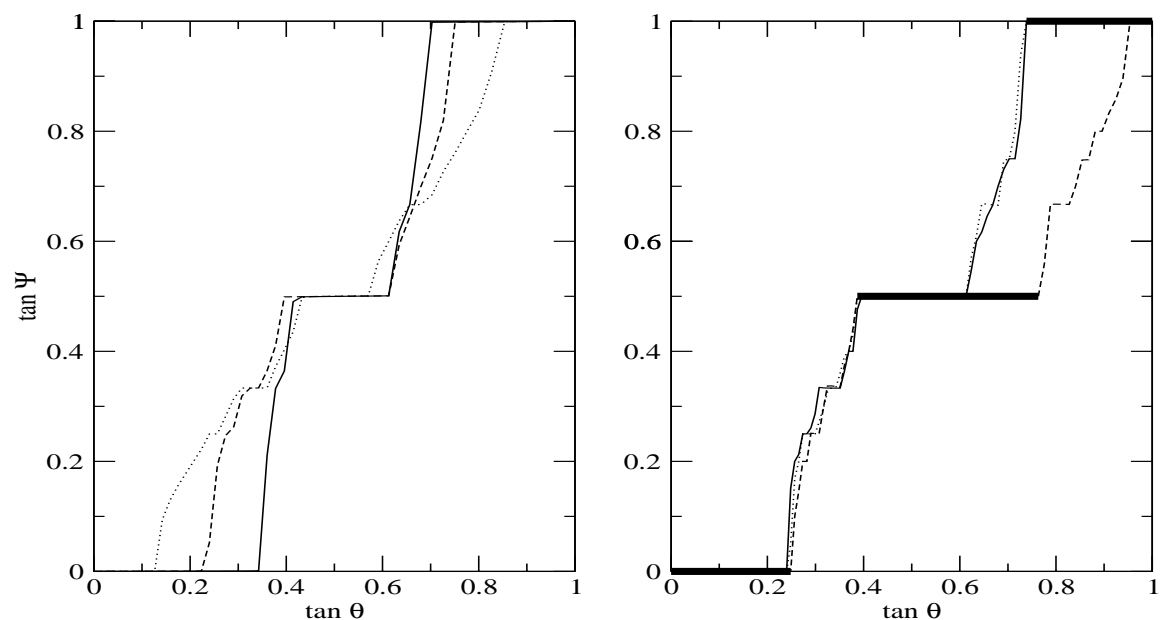

Fig. 3. Left panel: plateaus in the absolute velocity angle for a surface with traps with $B=0.5$ (solid), 0.7 (dashed), and 0.9 (dotted). Other parameters: $F_{0}=10$ and $\mathcal{T}=10^{-4}$. Right panel: comparison of plateaus for $B=0.7$ and $\mathcal{T}=0$ for wells (solid), obstacles (dotted) and mixed potential with $B_{W}=B_{O}=0.7$ (dashed). The mixed lattice supports a much wider middle plateau and narrower end plateaus than the other two lattices.

Early in the history of this subject perhaps the most dramatic (and surely not as clearly understood as it became later) observation was the occurrence of so-called "kinetically locked-in transport". ${ }^{2,3}$ When the velocity angle $\Psi$ is plotted against the 
force direction $\theta$, a pronounced series of plateaus along which $\Psi$ is independent of $\theta$ are seen [cf. Fig. 4 of Korda et al. ${ }^{3}$ ]. These plateaus are reproduced in our numerical simulations, ${ }^{16,17}$ and they occur for all the potentials discussed here. Examples are shown in Fig. 3. The plateaus are extreme manifestations of the point made in item 2 above. A fairly straightforward geometrical calculation of the conditions for these terraces at zero temperature is possible if we think of the surface as an array of cylindrical obstacles and/or traps of radius $r_{0}$. For instance, for an obstacle surface we construct the trajectories assuming that the particles go around the obstacles and move on along the line tangent to the cylinder and parallel to the direction of the force. In this case, if we take $r_{0}=0.9$ we readily find a plateu at $\Psi=0$ that extends over the range $\tan \theta \epsilon[0,0.23]$, one at $\Psi=0.5$ for the range $\tan \theta \epsilon[0.38,0.63]$, and a third at $\Psi=1$ for $\tan \theta \epsilon[0.72,1]$. The right panel of Fig. 3 shows that the angles $\Psi$ of the plateaus are insensitive to lattice geometry, and that the wells and obstacles give the same plateau widths whereas the mixed lattice leads to some narrower and some wider terraces. For instance, at the force angle $\tan \theta=0.5$ all three geometries have the particles following the angle $\tan \Psi=0.5$ but at the force angle $\tan \theta=0.75$ the wells and the obstacle surface trajectories lie along $\tan \Psi=1$ whereas those of the mixed lattice lie along $\tan \Psi=0.5$. For the cylindrical obstacle geometry we also find minor terraces located at $\tan \Psi=1 / 4,1 / 3,2 / 3,5 / 3$, and $3 / 4$.

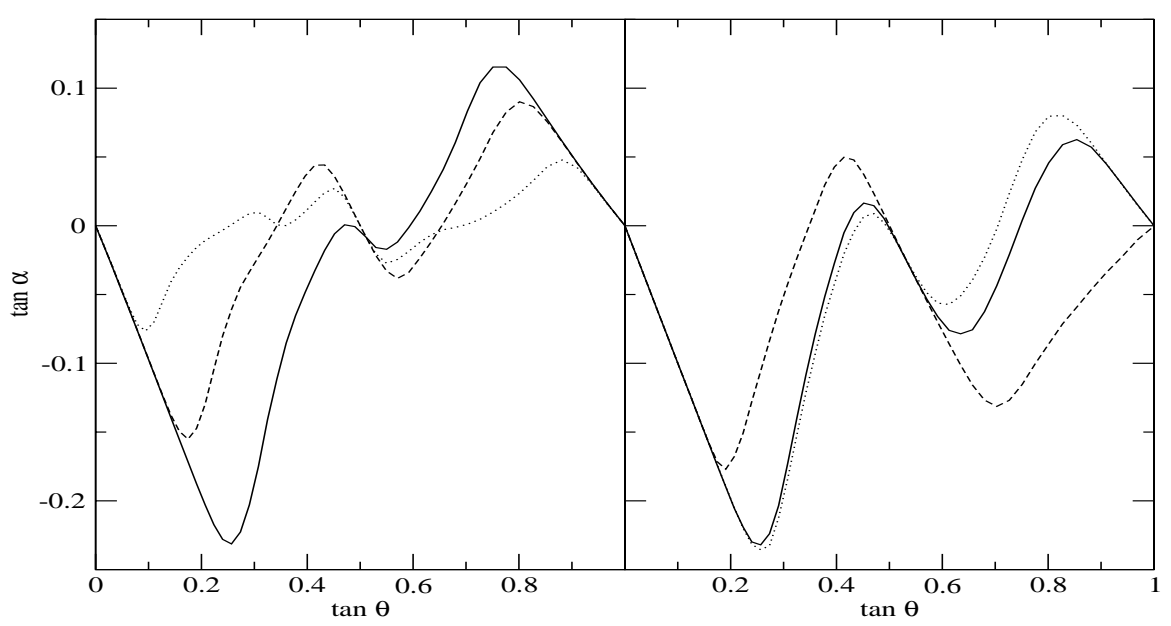

Fig. 4. Deflection angle vs force direction. Left panel: obstacles with $B=0.5$ (solid), $B=0.7$ (dashed) and $B=0.9$ (dotted). Right panel: mixed potential with $B_{W}=0.5$ and $B_{O}=0.9$ (solid), $B_{W}=0.7$ and $B_{O}=0.7$ (dashed), $B_{W}=0.9$ and $B_{O}=0.5$ (dotted). Other parameters: $F_{0}=10$, $\mathcal{T}=0.01$. 


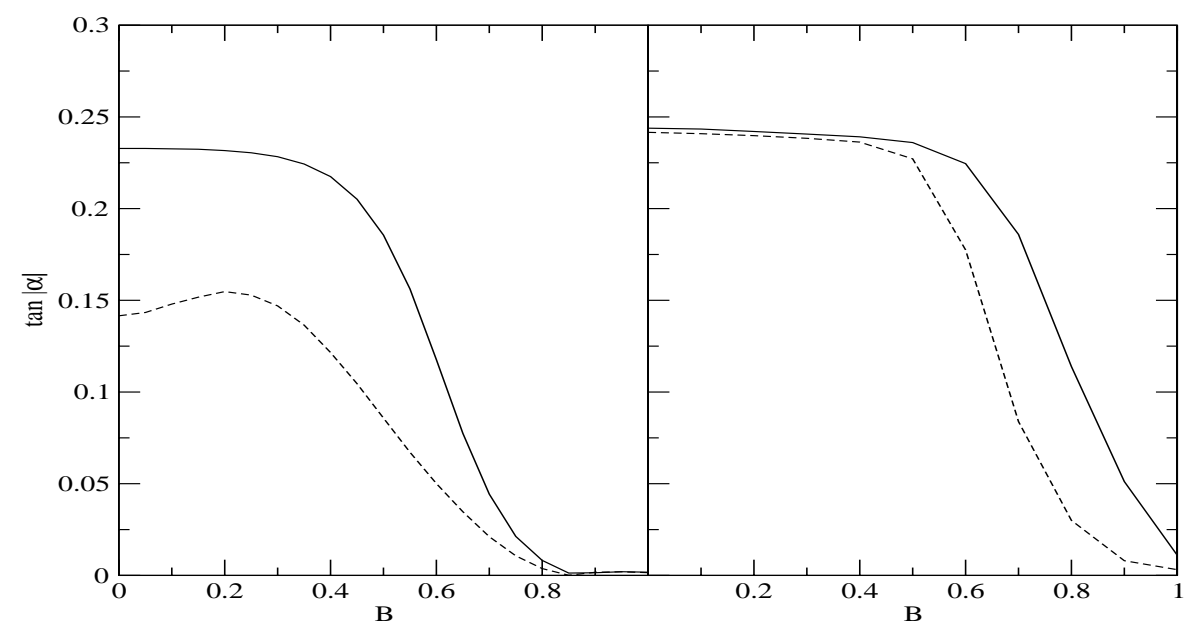

Fig. 5. Deflection angle vs particle size parameter $B$ for $\tan \theta=0.24$. Left panel: wells and $F=10$ (solid), $F=12$ (dashed). Right panel: obstacles and $F=4$ (solid), $F=10$ (dashed). For all cases $\mathcal{T}=0.01$.

A clear view of the sorting capabilities of the modulated surfaces is apparent if instead of the $\theta$ dependence of the velocity angle $\Psi$ we focus on the $\theta$ dependence of the deflection angle $\alpha$. Typical results for obstacles and mixed surface modulations are shown in Fig. 4. The results for wells (not shown) are essentially the same as for obstacles. Here we see clearly that sorting is possible because the deflection angle for a given force direction and magnitude depends on the size of the particles. The figure makes it clear that for each geometry one can in fact choose $\theta$ so as to optimize the sorting of particles of given sizes. For example, in the case of obstacles (left panel) sorting of particles corresponding to $B=0.5$ (solid) and $B=0.7$ (dashed) is optimized at a force angle $\tan \theta \sim 0.24$, while $B=0.7$ and $B=0.9$ (dotted) are most effectively sorted if one chooses $\tan \theta \sim 0.15$. Note the similarities between the surfaces with only obstacles (or only wells) and with wells and obstacles of different sizes as well as the considerable difference in the behavior of the mixed surface with equal and unequal $B$ values for $\tan \theta>0.5$. Overall, while the detailed values of the angles and of the sorting efficiency depend on the surface modulation, the same ideas are applicable in all cases.

In Fig. 5 we show the deviation angle as a function of the particle size parameter $B$ for a surface with wells and one with obstacles, each for two different forces (see Fig. 2C in Huang et al. ${ }^{9}$ ). In both cases a greater deviation angle for a given particle size is observed for lower forces. On the other hand, if a given deviation angle is sought, one must decrease the force for larger particles in the case of wells but one 
must increase the force for larger particles in the case of obstacles.

\section{Trajectories: dependence on $\boldsymbol{F}_{0}$}

We have described and illustrated the dependence of the trajectories of particles of different sizes on the direction of the external force, and have ascertained that one can choose the external force direction so as to optimize the sorting of particles of given different sizes. The detailed optimization depends on the specific surface modulation, but the fact that any periodic modulation can lead to sorting is clear. Next we direct our attention to the dependence of the particle trajectories on the magnitude of the external force.

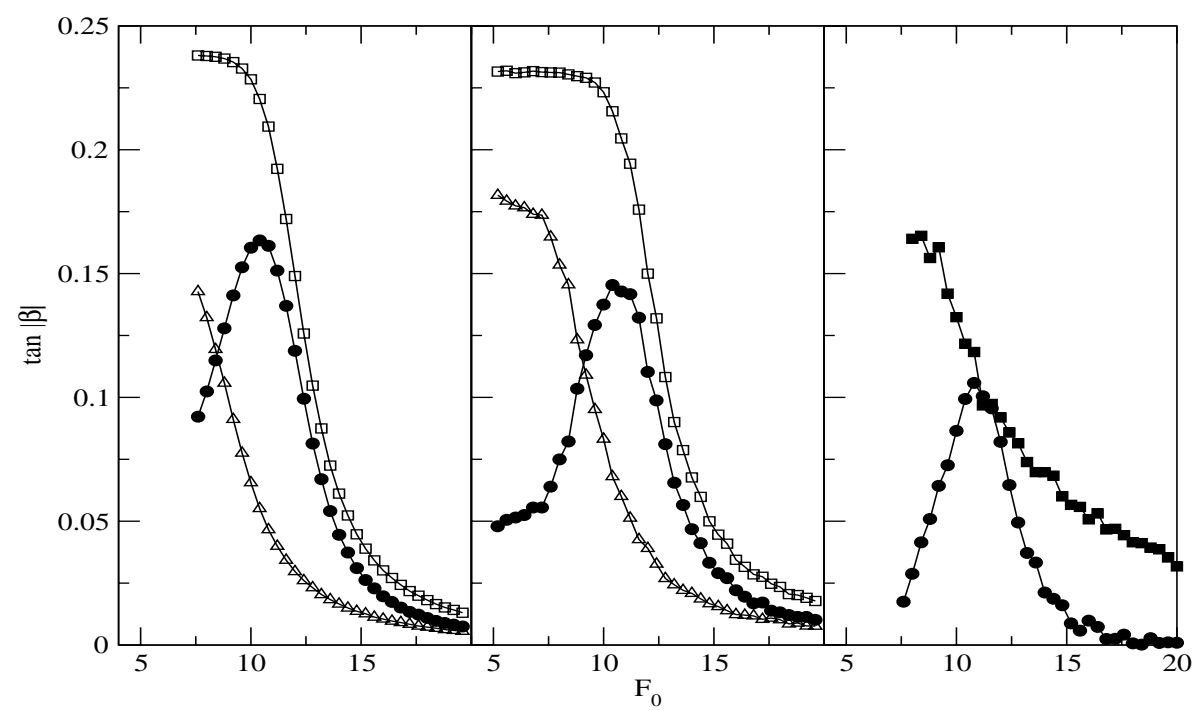

Fig. 6. Left (wells) and central (obstacles) panels: deflection angles $\alpha$ for $B=0.5$ (squares) and $B=0.7$ (triangles) for a force direction $\tan \theta=0.24$. The circles show the difference between the two deflection angles. Right panel: Difference in deflection angles for particles on a mixed surface $\left(B_{W}=0.5\right.$ and $\left.B_{W}=0.7, B_{O}=1.4-B_{W}\right)$ at two different force directions, $\tan \theta=0.24$ (circles) and $\tan \theta=0.8$ (squares). The larger angle is very effective for sorting; the difference in deflection angles exhibits no maximum within the range of forces of our simulations. Note that the optimal force at the angle $\tan \theta \sim 0.24$ is nearly the same for the three types of surfaces. In all cases $\mathcal{T}=0.01$.

It is clear from the outset that there must be a range of forces that is most useful for sorting. If the external force is too weak, the particles will become trapped in 
potential wells or will not be able to get around obstacles. If the force is too strong, the particles will essentially ignore the traps or obstacles and will simply follow the force. The range of forces for successful sorting lies between these two extremes, and, for appropriately chosen force directions, it is in fact possible to select an optimal force to achieve most efficient separation. This is shown in Fig. 6. On the vertical axes $\beta$ stands for either the deviation angle $\alpha$ for particles of a given size, or for the difference in deviation angles for particles of two sizes. This difference, which one seeks to optimize, is related to a commonly used "figure of merit" for sorting [see Fig. 3 of McDonald et al. ${ }^{7}$ ]. The force directions in the figure have been chosen on the basis of Fig. 4. We note one interesting result that points to some important differences among the surfaces due to geometry, and it is the fact that at a large angle (here $\tan \theta=0.8$ ) it is possible to sort particles very effectively at small external forces provided we have a mixed geometry. The other geometries do not exhibit this effectiveness at small forces.
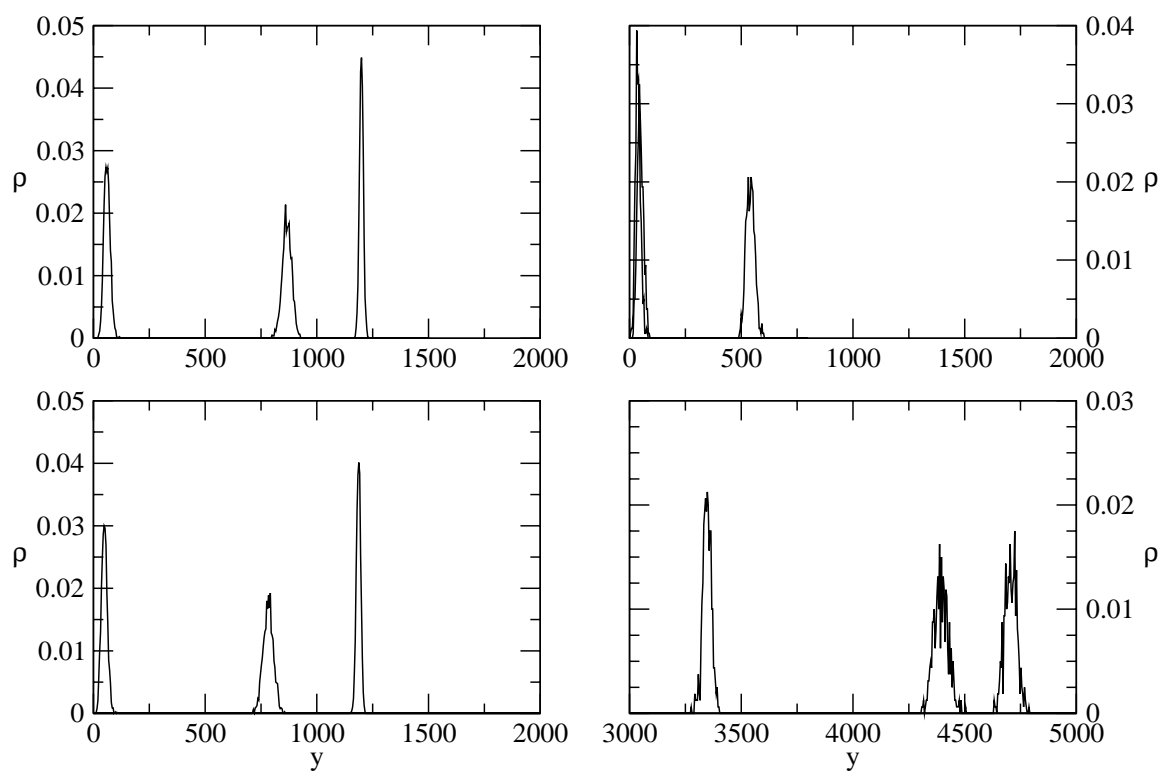

Fig. 7. $y$-position arrival distributions for three sets of 2000 particles, for $F=10$ and $\mathcal{T}=0.01$, collected at $L_{x}=5000$. Upper left (wells) and lower left (obstacles): $B=0.5,0.7$, and 0.9 (left to right), $\tan \theta=0.24$. Upper right: mixed lattice with $B_{W}=0.5,0.7$, and 0.9 (left to right), $B_{O}=1.4-B_{W}$, and $\tan \theta=0.24$. Lower right: same mixed lattice but $\tan \theta=0.8$. 
While the difference in deflection angles is a crucial measure of the sorting capability of a modulated surface, one should also take into account the dispersion of the particles as they reach the end of the system. If this dispersion is too large, e.g., if the particle distribution were to be too broad around the deflection angle so that the distributions of different mean deflection angles overlap, then a measure based only on these averages may not be sufficient for a good selection process. In Fig. 7 we show the distributions associated with the deflection angle results in Fig. 6 . The vertical axes show the distributions. That the particles of different sizes for wells and for obstacles emerge at different angles when the external force angle is $\tan \theta=0.24$ not only agrees with the results of Fig. 6, but also with those of Fig. 4. However, this angle is not of use to separate $B=0.5$ from 0.9 in the mixed case, in agreement with the right panel of Fig. 4 . However, $\tan \theta=0.8$ is a good separation angle for all three particle sizes in the mixed case. Clearly, for all cases illustrated in this figure the mean deflection angles do provide a good indicator for sorting. These results provide a useful additional measure for comparison with experiments. ${ }^{10}$ Given a set of particles of two different sizes, the resolution $R$ is calculated from the $y$-position distributions for the two types of particles (collected at a fixed $x$-position) as

$$
R=\frac{\Delta Y}{2 \sigma_{1}+2 \sigma_{2}},
$$

where $\Delta Y$ is the peak separation and $2 \sigma_{i}$ are the bandwiths. Resolvable distributions are associated with values of $R \gtrsim 1 .{ }^{18}$ The resolution for the deflection angles and associated distributions shown in Figs. 6 and 7 are shown in Fig. 8. The maxima in the resolution curves indicate the optimal force for sorting of particles of those particular sizes and force directions.

\section{Other Variations}

There are two quantities that we have essentially kept fixed in our presentation (or varied very little), namely, the temperature and the potential parameter $A$. In this section we briefly review some consequences of varying these quantities.

Raising the temperature necessarily blurs the sorting mechanism. We illustrate this obvious result in a variety of ways using the measures that we have introduced in previous sections. First, consider the plateaus in the absolute velocity angle as a function of the direction of the external force, as illustrated in Fig. 3. Raising the temperature blurs these sharp terraces, as shown in the left panel of Fig. 9. The $y$-distribution of particles upon arrival at a particular $L_{x}$, as illustrated in the right panel of Fig. 7, of course depends on temperature and also blurs with increasing temperature. An example is shown in the right panel of Fig. 9. The lowest $\mathcal{T}$ case here is the same as the leftmost panel of Fig. 7. The associated progression of the resolution is shown in Fig. 10.

Finally, the one remaining parameter that we have not varied in this discussion is the parameter $A$ in the surface modulation potential. As noted earlier, this parameter allows us to control the steepness of the wells and/or obstacles and therefore the 


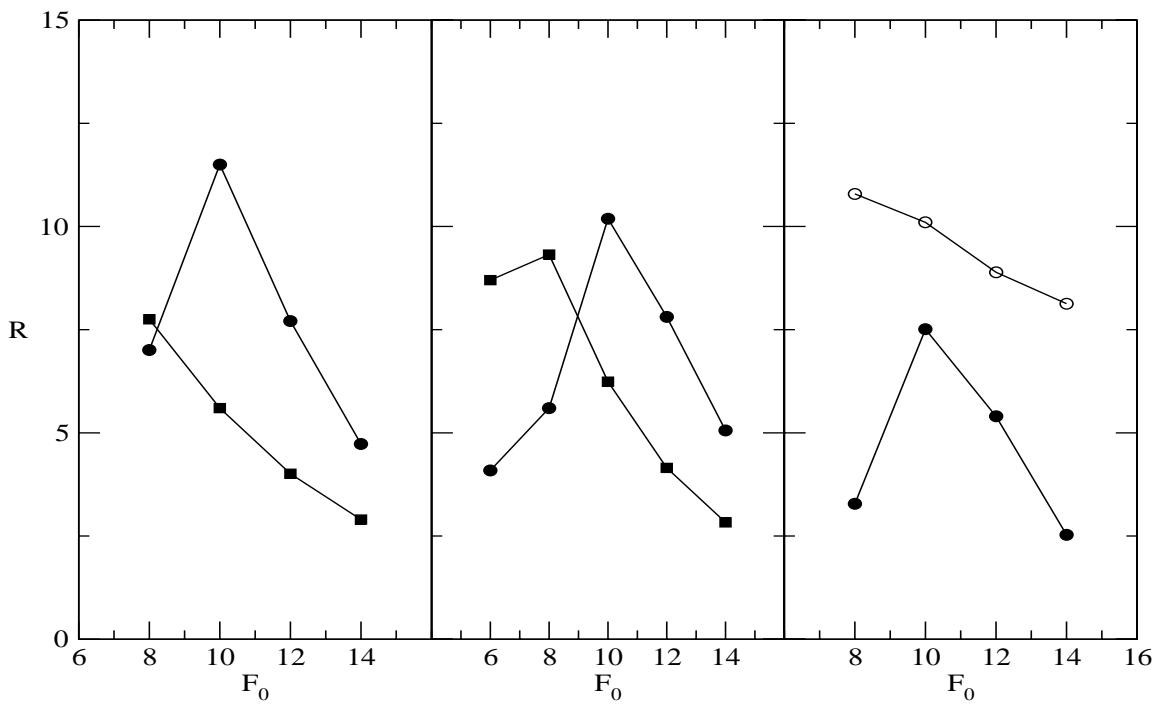

Fig. 8. Resolution as a function of the magnitude of the external force for particles of two different sizes at $\mathcal{T}=0.01$. Left (wells) and middle (obstacles) panel: $B=0.5$ and 0.7 (circles), $B=0.7$ and 0.9 (squares), and $\tan \theta=0.24$. Right panel: mixed lattice with $B_{W}=0.5$ and $0.7, B_{O}=1.4-B_{W}$, and $\tan \theta=0.24$ (solid circles), $\tan \theta=0.8$ (open circles).

size of the flat regions connecting them. In the mixed potential case, we indicated that a judicious choice of $A$ allows us to mimic even a purely cosinusoidal potential within the family of potentials Eqs. (9)-(11). In Fig. 11 we show the deflection angle $\alpha$ as a function of the direction of the external force for a mixed lattice with various values of $A$, other parameters being held fixed. The figure shows that $A$ can have a considerable effect on the detailed sorting behavior of the system, and that it is indeed possible to use our potential to mimic the behavior of the purely cosinusoidal potential

$$
V(x, y)=-\frac{V_{0}}{2}\left[\cos \left(\frac{2 \pi x}{\lambda}\right)+\cos \left(\frac{2 \pi y}{\lambda}\right)\right] .
$$

Elsewhere we have investigated sorting in purely harmonic potentials, both symmetric and asymmetric $\left(\lambda_{x} \neq \lambda_{y}\right)$, and have established that deviation angles in these potentials are larger than in those with only wells or only obstacles, all other parameters being the same. ${ }^{17}$ Here we have extended the model to include wells and obstacles as in a purely harmonic potential, but with the ability to further calibrate the heights, depths, and widths of the wells and obstacles. 

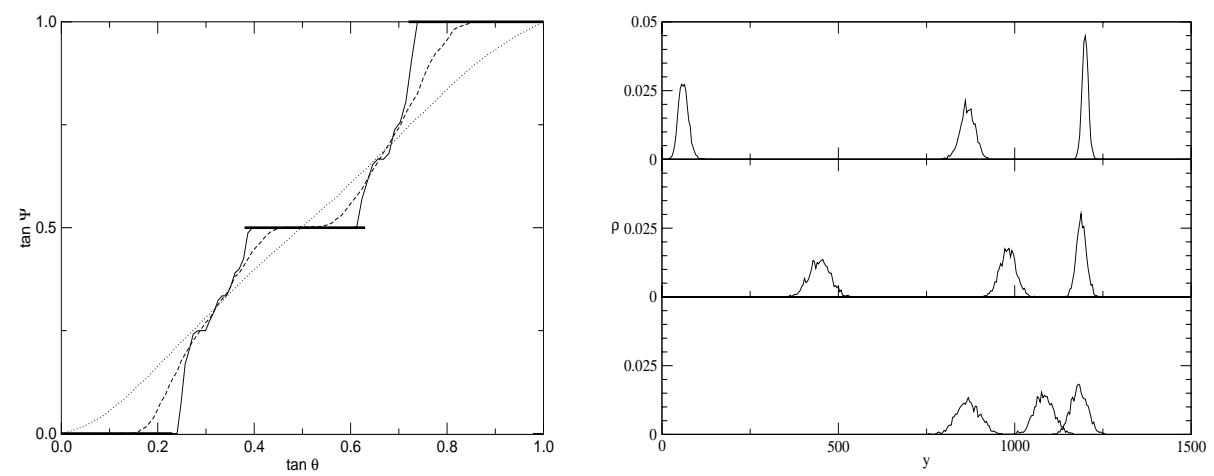

Fig. 9. Left panel: effect of thermal fluctuations on the terraces in the absolute angle as a function of the direction of the external force. In this example the lattice is one of obstacles with $B=0.7$ and $T=10^{-4}$ (solid, same as shown in upper right panel of Fig. 3), $T=0.01$ (dashed), and $T=0.1$ (dotted). The terraces are essentially gone at the highest temperature. Right panel: $y$ position arrival distributions for three sets of 2000 particles corresponding to $B=0.5,0.7$ and 0.9 (from left to right) in an array of wells. Particles are collected at $L_{x}=5000$. Upper: $\mathcal{T}=0.01$. Middle: $\mathcal{T}=0.03$. Lower: $\mathcal{T}=0.1$. Other parameters are: $F_{0}=10$ and $\tan \theta=0.24$.

\section{Conclusions}

When mixtures of particles are made to flow over periodic modulated surfaces under appropriate conditions, the particles emerge at different angles that depend on some particle characteristic such as size. Mixtures can therefore be sorted in this way. In an experiment the particle characteristics of a mixture are of course fixed, and so one seeks the direction and magnitude of the flow and the lattice geometry that will optimize the sorting process. We have presented an extensive panorama of numerical results for particle sorting by size using surfaces consisting of periodic distributions of traps and of periodic distributions of obstacles (both of which have been realized experimentally for colloid separation ${ }^{3-10,14}$ ), and of trap and obstacle mixtures. We have discussed the dependence of particle trajectories on the various parameters and on the temperature, and have demonstrated that parameters can be chosen so as to optimize the sorting efficiency. While the detailed outcome depends on the surface geometry, the sorting capability is a general feature of any periodic modulation.

Embellishments of the models are of course plentifully possible. One could consider lattices of different symmetries (which we have done earlier for purely cosinusoidal lattices ${ }^{17}$ ). The surface structures could be more elaborate than the circular ones considered here. For instance, experiments have been carried out with rectangular obstacles ${ }^{9}$ and inclined obstacles ${ }^{10}$ as well as with optical line tweezers ("optical travelators"). ${ }^{14}$ It would be desirable to develop analytic approaches beyond the perturbative ones that we have presented elsewhere. ${ }^{15}$ Finally, it would be interesting to understand the flow of particles over random surfaces. Our analytic 


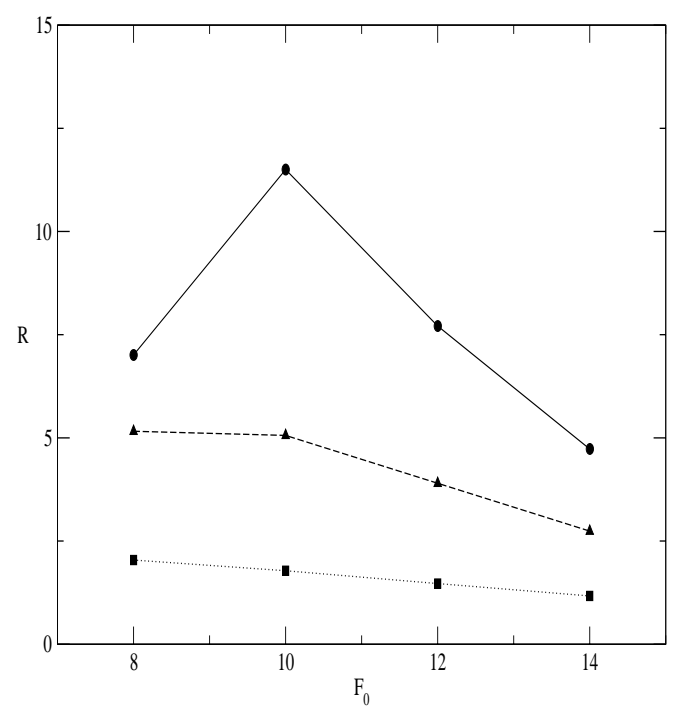

Fig. 10. Resolution associated with the distributions shown in Fig. 9 for wells as a function of the force at $\tan \theta=0.24$ for particles of two different sizes corresponding to $B=0.5$ and $B=0.7 . \mathcal{T}=0.01$ (circles), $\mathcal{T}=0.03$ (triangles), and $\mathcal{T}=0.1$ (squares). $R \gtrsim 1$ is experimentally resolvable. ${ }^{18}$

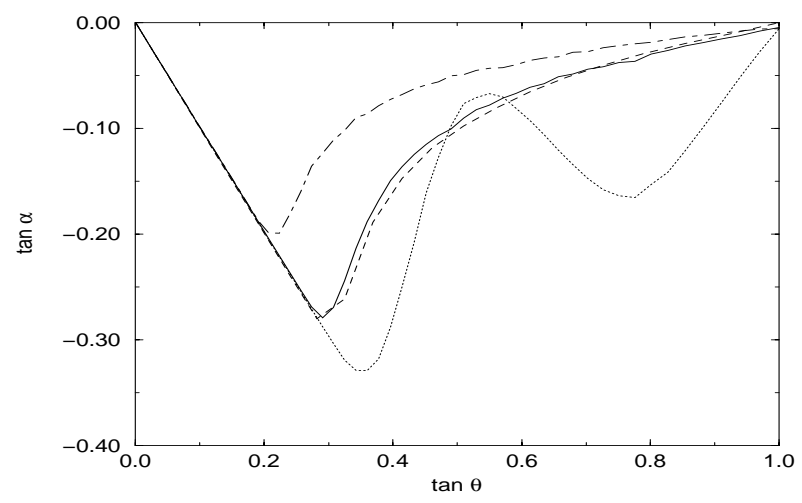

Fig. 11. Deflection angle versus field direction for $F_{0}=10, \mathcal{T}=0.01, B_{W}=B_{0}=0.5$ and parameter $A=1$ (dot-dashed), $A=2$ (solid) and $A=5$ (dotted). In addition, results for the harmonic periodic potential (16) are also shown (dashed) for the same values of $F_{0}$ and $\mathcal{T}$.

work $^{15}$ (within the limits of perturbation theory) indicates that sorting over such surfaces is possible if they are not isotropic. 


\section{Acknowledgements}

This work was supported by the MCyT (Spain) under project FIS2006-11452 and by the National Science Foundation under grant No. PHY-0354937.

\section{References}

1. A. J. Duke and R. H. Austin, Phys. Rev. Lett. 80, 1552 (1997).

2. C. Reichhardt F. Nori, Phys. Rev. Lett. 82, 414 (1999); C. Reichhardt and C. J. Olson Reichhardt, Phys. Rev. E 69, 041405 (2004); Europhys. Lett. 68, 303 (2004).

3. P. T. Korda, M. B. Taylor, and D. G. Grier, Phys. Rev. Lett. 89, 128301 (2002).

4. K. Dholakia, G. Spalding, and M. MacDonald, Physics World, October, 31 (2002).

5. D. G. Grier, Nature 424, 810 (2003).

6. A. Gopinathan and D. G. Grier, Phys. Rev. Lett. 92, 130602 (2004).

7. M. P. MacDonald, G. C. Spalding, and K. Dholakla, Nature 426, 421 (2003).

8. D. G. Grier and Y. Roichman Appl. Opt. 45, 880 (2006).

9. L. R. Huang, E. C. Cox, R. H. Austin, and J. C. Sturm, Science 304, 987 (2004).

10. L. R. Huang, E. C. Cox, R. H. Austin, and J. C. Sturm, Analytical Chemistry 75, 6963 (2003).

11. K. Ladvac, K. Kasza, and D. G. Grier, Phys. Rev. E 70, 010901 (2004).

12. M. Pelton, K. Ladavac, and D. G. Grier, Phys. Rev. E., 70, 031108 (2004).

13. S. Savel'ev, V. Misko, F. Marchesoni and F. Nori, Phys. Rev. B 71, 214303 (2005).

14. F. C. Cheong et al., Appl. Phys. B 83, 121 (2006).

15. J.P. Gleeson, J.M. Sancho, A.M. Lacasta and K. Lindenberg, Phys. Rev. E 73041102 (2006).

16. A. M. Lacasta, J. M. Sancho, A. H. Romero, and K. Lindenberg, Phys. Rev. Lett. 94, 160601 (2005).

17. J.M. Sancho, M. Khoury, K. Lindenberg and A.M. Lacasta, J. Phys: Condens. Matter 17, S4151 (2005).

18. J. C. Giddings, Unified Separation Science (John Wiley and Sons, New York, 1991), pg. 101. 